\title{
Protozoários gastrointestinais em Tayassu pecari mantidos em cativeiro no Brasil
}

\section{Gastrointestinal protozoa in Tayassu pecari kept in captivity in Brazil}

\author{
Matheus Hilliard Farret' ${ }^{1}$; Vinícius da Rosa Fanfa ${ }^{1}$; \\ Aleksandro Schafer da Silva ${ }^{2}$; Silvia Gonzalez Monteiro ${ }^{3 *}$
}

\section{Resumo}

Este estudo teve como objetivo registrar o parasitismo gastrintestinal em queixada (Tayassu pecari) no Brasil. Foram colhidas amostras de fezes, de três exemplares mantidos em cativeiro no estado do Rio Grande do Sul. As amostras foram analisadas pelas técnicas de centrífugo flutuação com sulfato de zinco e coloração de kinyon para pesquisa de parasitos. Em todos os animais observou-se uma infecção mista por cistos de Giardia sp. e Balantidium sp., além de oocistos de Cryptosporidium sp e Eimeria sp. Este estudo relata o primeiro registro destes quatro gêneros de protozoários em T. pecari.

Palavras-chave: Giárdia, Cryptosporidium, Eimeria, Balantidium

\begin{abstract}
The aim of this study was to report the gastrointestinal parasitism in white-lipped peccary (Tayassu pecari) in Brazil. Fecal samples of three animals kept in captivity in the state of Rio Grande do Sul were collected. Samples were analyzed by the centrifugal flotation technique with zinc sulfate and by the Kinyon staining method. All animals showed mixed infection by cysts of Giardia sp. and Balantidium sp. and by oocysts of Cryptosporidium sp. and Eimeria sp. This is the first report of these four genera of protozoa in T. pecari.
\end{abstract}

Key words: Giardia, Cryptosporidium, Eimeria, Balantidium

\footnotetext{
${ }^{1}$ Curso de Medicina Veterinária da Universidade Federal de Santa Maria - UFSM, Santa Maria. E-mail: mfarret@superig.com.br

${ }^{2}$ Pós graduação em Medicina Veterinária da Universidade Federal de Santa Maria - UFSM. E-mail: aleksandro_ss@yahoo.com. br

${ }^{3}$ Docente do Departamento de Microbiologia e Parasitologia da Universidade Federal de Santa Maria - UFSM. Faixa de Camobi - Km 9, Campus Universitário, Santa Maria - RS, Brasil. 97105 900, prédio 20, sala 4232. fax: (55) 3220-8958. E-mail: sgmonteiro@uol.com.br

* Autor para correspondência
} 
Tayassu pecari (queixada) é um mamífero da família Tayassuidae, pertencente à ordem Artiodactyla que é formada por três espécies, Tayassu tajacu, Tayassu pecari e Catagonus wagneri. Os exemplares da família em questão são também conhecidos como porcos do mato, porém não pertencem a família Suidae que é representada pelo porco doméstico e o javali (SILVA, 2006). T. pecari é o maior dos porcos selvagens, o qual pode chegar a 1,10 m de comprimento. Podem ser encontrados desde o sul do México até o nordeste da Argentina (DEUTSCH; PUGLIA, 1990).

Em T. pecari já foi reportado o parasitismo por vários ectoparasitos e endoparasitos, entre eles carrapatos das espécies Amblyomma cajennense, Amblyomma inornatum e Amblyomma pecarium e helmintos das espécies Macracanthorhynchus hirudinaceus, Physocephalus sexalatus, Ascarops strongylina, Trichostrongylus axei, Texicospirura turki, Spiculopteragia tayassui, Parostertagia heterospiculum, Monodontus semicircularis, Monodontus aguiari, Eucyathostomum dentatum, Oesophagostomum dentatum, Stichorchis giganteus, Dirofilaria acutiúscula, Metastrongylus salmi, Bunostomum sp., Parabronema pecariae, Paramphistomum sp. e Moniezia benedeni (NASCIMENTO; HOPPE; MAPELI, 2005; CUBAS; SILVA; CATÃO-DIAS, 2006; ROMEROCASTAÑÓN et al., 2008).

Como citado anteriormente, pesquisas reportam vários helmintos em queixada (T. pecari), porém há uma carência nas pesquisas de protozoários nestes mamíferos. Outro ponto relevante é o papel destes animais silvestres na disseminação de parasitos com potenciais zoonóticos (FAYER; MORGAN; UPTON, 2000). Visto isto, este trabalho relata pela primeira vez quatro protozoários gastrintestinais em queixada mantidos em cativeiro no sul do Brasil.

Exames de rotina são realizados em animais silvestres de um criadouro conservacionista na região central do estado do Rio Grande do Sul. Entre os mamíferos avaliados havia três exemplares adultos, duas fêmeas e um macho de T. pecari. As amostras foram armazenadas refrigeradas, em temperatura de $13{ }^{\circ} \mathrm{C}$, por 24 horas. As mesmas foram processadas pelas técnicas de centrífugo flutuação e kinyon para pesquisa de parasitos (DE CARLI; MOURA, 2000). Os oocistos de coccídeos foram mantidos em temperatura e umidade controlada $\left(27^{\circ} \mathrm{C}\right.$ e $80 \%$ UR $)$ para esporulação a fim de identificação do gênero de acordo com a morfologia e tamanho dos cistos, oocistos e esporocistos (KUDO, 1886; HOFFMAN, 1987; FAYER; MORGAN; UPTON, 2000). O grau de infecção foi classificado de acordo com Atlantic Veterinary College University of Prince Edward Island (2007).

Observou-se em todas as amostras a presença de infecção mista por cistos de Giardia sp. e Balantidium sp., e oocistos de Cryptosporidium sp. e Eimeria sp. (Figura 1). Na coloração de kinyon confirmouse a presença de Cryptosporidium sp. Os animais apresentaram infecção leve pelos protozoários (10-46 cistos/oocistos/lâmina) e não manifestaram sinais clínicos decorrentes da doença. Este achado trata-se do primeiro registro de parasitismo misto por Giardia sp., Cryptosporidium sp, Eimeria sp. e Balantidium sp. em queixada. Os cistos de Giardia sp. apresentaram variação de tamanho entre $8-11$ e $9-12 \mu \mathrm{m}$. Os oocistos de Cryptosporidium sp. mediram em média 5-6 $\mu \mathrm{m}$. O tamanho dos oocistos de Eimeria sp. oscilaram entre $19-23$ e $20-24 \mu \mathrm{m}$ e os esporocistos mediaram em media $6-7 \mu \mathrm{m}$. Os cistos de Balantidium sp. mediram entre $31-34$ e $33-35 \mu \mathrm{m}$.

Entre os parasitos identificados destacam-se Giardia sp. e Cryptosporidium sp., agentes causais de duas zoonoses em potencial, que geralmente causam doença clínica em animais jovens, porém, podem ser encontrados em animais adultos (CUBAS; SILVA; CATÃO-DIAS, 2006), como neste estudo. Estes protozoários já foram relatados causando infecção em seres humanos, cães, gatos, bovinos, suínos, ovinos, eqüinos e animais silvestres (FAYER; MORGAN; UPTON, 2000; LALLO; BONDAN; RODRIGUES, 2003; CUBAS; SILVA;CATÃO-DIAS, 2006). 
Acredita-se que a carência de investigação na área de silvestres ou o uso de técnicas pouco específicas para protozoários seja a causa destes parasitos não terem sido reportados anteriormente.

Cateto (Tayassu tajacu), parente próximo do queixada, foram descritas quatro espécies do gênero Eimeria (WILBER; HELLGREN; GABOR, 1996) e infecção por Balantidium coli (SCHETTINI; LIE; GALVEZ, 2005). Estes dois protozoários são comuns em granjas de suínos e criações de javalis (NISHI et al., 2000; MUNDIM et al., 2004; HOFF; SILVA; MONTEIRO, 2005). Com o aumento das restrições dos órgãos ambientais, cresceu o número de animais silvestres reproduzidos em cativeiro, dentre eles os da família Tayassuidae, visto isso se considera importante conhecer as parasitoses que podem acometer os queixadas a fim de tratamento e profilaxia.

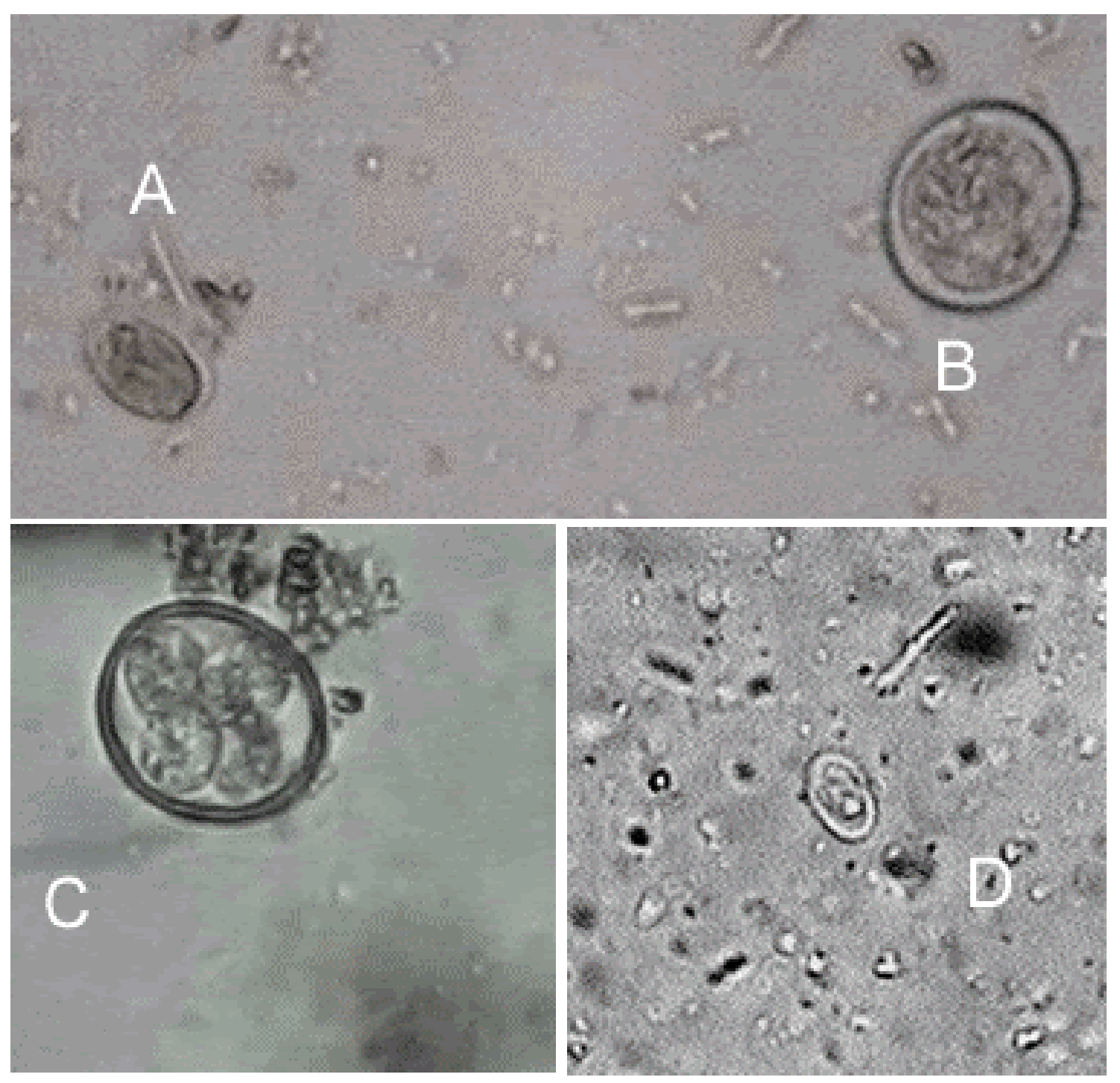

Figura 1. Formas imaturas de parasitos encontrados em amostras de fezes de Tayassu pecari mantido em cativeiro: (A) cistos de Giardia sp.; (B) cistos de Balantidium sp.; (C) oocistos de Eimeria sp.; (D) oocistos de Cryptosporidium sp. 
Com o aumento de pesquisas na área de parasitologia da fauna silvestre tem se conhecido novos hospedeiros para diferentes agentes etiológicos como foi observado neste estudo. O $T$. pecari é um hospedeiro em potencial para os gêneros Giardia, Cryptosporidium, Eimeria e Balantidium. $\mathrm{O}$ fato dos animais com infecção mista por estes protozoários, não apresentarem sinais clínicos da doença, possivelmente deve-se a baixa infecção, ou somente, pelo fato de serem reservatórios e disseminadores destes parasitos.

\section{Referências}

ATLANTIC Veterinary College University of Prince Edward Island. Diagnostic services: parasitology. 2007. Disponível em:< http://www.upei.ca/diagserv/html/ parasitology.html>. Acesso em: 17 jul. 2008.

CUBAS, Z. S.; SILVA, J. C. R.; CATÃO-DIAS, J. L. Tratado de animais silvestres. São Paulo: Roca, 2006. $1354 \mathrm{p}$.

DE CARLI, G. A.; MOURA, H. Parasitologia clínica: diagnóstico de laboratório dos coccídeos e microsporídios intestinais. Porto Alegre: EDIPUCRS, 2000. 73 p.

DEUTSCH, L. A.; PUGLIA, L. R. Os animais silvestres - proteção, doenças e manejo. 2. ed. São Paulo: Globo, 1990. $191 \mathrm{p}$.

FAYER, R.; MORGAN, U.; UPTON, S. J. Epidemiology of Cryptosporidium: transmission, detection and identification. International Journal for Parasitology, Austrália, v. 30, n. 1, p. 12-13, 2000.

HOFF, G.; DA SILVA, A. S.; MONTEIRO, S. G. Avaliação do parasitismo e comparação de técnicas de análise fecal em suínos de granjas da região oeste do estado de Santa Catarina. Revista da Faculdade de Zootecnia, Veterinária e Agronomia, Uruguaiana, v. 12, n. 1, p. 20-30, 2005.

HOFFMAN, R. P. Diagnóstico deparasitismo veterinário. Porto Alegre: Sulina, 1987. 156 p.

KUDO, R. R. Protozoology. Illinois: Charles C Thomas, Publisher, 1886. 966 p.
LALLO, M. A.; BONDAN, E. F.; RODRIGUES, L. C. S. Giardíase em cães e gatos. Clínica Veterinária, São Paulo, v. 43, n. 1, p. 40-46, 2003.

MUNDIM, M. J. S.; MUNDIM, A. V.; SANTOS, A. L. Q.; CABRAL, D. D.; FARIA, E. S. M.; MORAES, F. M. Helmintos e protozoários em fezes de javalis (Sus scrofa scrofa) criados em cativeiro. Arquivo Brasileiro de Medicina Veterinária e Zootecnia, Belo Horizonte, v. 56, n. 6, p. 792-795, 2004.

NASCIMENTO, A. A.; HOPPE, E. G.; MAPELI, E. B. Infecções naturais por helmintos parasitas em queixadas (Tayassu Pecari) no pantanal de paiaguás, MS, Brasil. Revista de Patologia Tropical, Goiânia, v. 34, p. 115-116, 2005. Suplemento 1.

NISHI, S. M.; GENNARI, M. N. T. S.; LISBOA, A.; SILVESTRIM, L.; CAPRONI, J. R.; UMEHARA, O. Parasitas intestinais em suínos confinados nos estados de São Paulo e Minas Gerais. Arquivo do Instituto de Biológico, São Paulo, v. 67, n. 2, p. 199-203, 2000.

ROMERO-CASTAÑÓN， S.; FERGUSON， B. G.; GÜIRIS, D.; GONZÁLEZ, D.; LÓPEZ, S.; PAREDES, A.; WEBER, M. Comparative parasitology of wild and domestic ungulates in the selva Lacandona, Chiapas, México. Comparative Parasitology, Flórida, v. 75, n. 2, p. 115-126, 2008.

SChetTINI, Z. L.; LIE, O.; GALVEZ, C. H. Perfil bioquímico sanguíneo hepático y renal en el sajino (Tayassu tajacu) criado en cautiverio en la amazonía peruana. Revista de Investigaciones Veterinarias del Peru, San Barja, v. 16, n. 2, p. 175-179, 2005.

SILVA, R. W. Avaliação da variabilidade genética em Tayassu tajacu (cateto) e Tayassu pecari (queixada) por meio da utilização de marcadores microssatélites. 2006. Disponível em: <http://genetica.bio.ufpr.br/ posgraduacao/teses/roxane.pdf $>$. Acesso em: 10 jul. 2008.

WILBER, P. G.; HELLGREN, E. C.; GABOR, T. M. Coccidia of the collared peccary (Tayassu tajacu) in southern Texas with descriptions of three new species of Eimeria (Apicomplexa: Eimeriidae). Journal of Parasitology, Lancaster, v. 82, n. 4, p. 624-629, 1996. 